(range: $6 \mathrm{~m}-47.5 \mathrm{y}$ ). Most troublesome symptoms at assessment were bowel frequency of 4 or more times a day $(88 \%)$, type 6 or 7 stool consistency (36\%), urgency to defaecate (31\%), faecal incontinence (21\%) and fatigue (25\%). Some women also reported urinary problems $(17 \%)$ and sexual concerns $(12 \%)$. Following a published algorithm, a median of 8 investigations were requested (range: 1-14): blood screen (97\%), gastroscopy (75\%), glucose hydrogen methane breath test (77\%), SeHCAT scan (71\%), faecal elastase (69\%), flexible sigmoidoscopy (52\%), colonoscopy (25\%) and abdominal Xray (18\%). A median of four factors contributing to symptoms were found (range: 1-9) and 68\% of women had more than three. The most common diagnoses were vitamin $\mathrm{D}$ deficiency (60\%), treated with replacement; SIBO (54\%), treated with antibiotics and bile acid malabsorption (52\%), treated with bile acid sequestrants and low fat diet. The median number of consultations was 4 (range: 1-17). Median quality of life improved from 4 at first assessment to 6 at discharge $(p<0.001)$; the reduction in urgency, incontinence, tenesmus, frequency, diarrhoea and fatigue was statistically significant $(\mathrm{p}<0.05)$.

Conclusions Bowel symptoms after cancer treatment affect many women. They can be discharged with symptom improvement using a systematic investigational and treatment approach. Earlier referral to specialist services is recommended.

\section{PTU-119 UPPER GASTROINTESTINAL HAEMORRHAGE: IS THERE A WEEKEND EFFECT? A REVIEW OF TWO DISTRICT GENERAL HOSPITALS}

${ }^{1}$ Duncan Napier* ${ }^{1}$ Rebecca Anderson, ${ }^{1}$ Giovanna Sheiybani, 2,35tephen Roberts, 2,3John Williams, 1,3 Jonathan Brown. 'Gloucestershire Hospitals NHS Foundation Trust, Gloucester, UK ${ }^{2}$ Swansea University Medical School, Swansea, UK ${ }^{3}$ Farr Institute of Health Informatics Research, Swansea University, Swansea, UK

\subsection{6/gutjnl-2018-BSGAbstracts.497}

Introduction Upper gastrointestinal haemorrhage (UGIH) is a common emergency presentation with a mortality reaching $10 \%{ }^{1}$ A 'weekend effect' has been described for UGIH with increased mortality rates for those admitted over a weekend. ${ }^{2}$ These studies typically utilise information from national databases to describe this effect whereas this study sought to examine if there was a reproducible 'weekend effect' at two district general hospitals.

Methods Retrospective data was extracted from the endoscopy database for both hospitals in 2014, identifying all patients with an indication suggestive of UGIH. The Trust coding database was used to identify all patients with an ICD-10 code suggestive of UGIH. These datasets were amalgamated and electronic admission records subsequently analysed to exclude inpatient UGIH. Admission and discharge documentation, endoscopy reports, GP records and bereavements records were reviewed to confirm day and time of admission and endoscopy, and survival to 30 days. Chi-squared test was used to compare mortality between groups.

Results There were 552 admissions for acute UGIH in 2014, 518 patients underwent an emergency endoscopy, 23 either did not have an endoscopy or had an outpatient endoscopy, and 11 notes were unavailable or incomplete and thus excluded. There was no statistically significant difference in 30 day mortality for those admitted on a weekday (Mon 0000 - Fri 2359) vs a weekend (11.05\% CI 7.98-14.79 vs
12.23\% CI 7.92-17.79, $\mathrm{p}=0.68 \mathrm{X}^{2}$ ). Neither was there a statistically significant difference in 30 day mortality for those admitted out of hours (1700-0859) compared to in hours (12.60\% CI $8.83-17.23$ vs $10.39 \%$ CI $7.07-14.59 \quad \mathrm{p}=0.43$ $\mathrm{X}^{2}$ ). Although not statistically significant, there was an increase in 30 day mortality for those requiring an out of hours procedure (1800-0759) compared to day time (23.08\% CI $14.89-33.09$ vs $8.64 \%$ CI $\quad 6.16-11.72 \quad \mathrm{p}=0.19$ $\mathrm{X}^{2}$ ).

Conclusions This study found no correlation between the day or time of admission for UGIH and 30 day mortality, suggesting that despite reduced levels of staffing and endoscopic activity over the weekend or out of hours this had no impact on 30 day mortality. This may be explained by appropriate patient selection for urgent endoscopy.

\section{PTU-120 IRON DEFICIENCY ANAEMIA IN RENAL CELL CARCINOMA}

Jeremy Nayagam*, Sun Ha, Lenka Barreto, Angelika Zang, Howard Curtis. Princess Royal University Hospital, King's College NHS Foundation Trust, London, UK

\subsection{6/gutjnl-2018-BSGAbstracts.498}

Introduction British Society of Gastroenterology (BSG) iron deficiency anaemia (IDA) investigation guidelines recommend urinalysis to screen for non-visible haematuria. If detected, renal ultrasound is recommended to exclude renal cell carcinoma (RCC). Data on IDA in RCC however suggests that haematuria is rarely detected. We sought to identify the prevalence of IDA in RCC, and the frequency of haematuria in this subgroup.

Methods All newly diagnosed RCC at a single NHS Trust over a 3 year period (1/1/13-31/12/15) were identified from a prospective database of the Urology multidisciplinary meeting (MDM). A retrospective analysis of electronic notes, MDM outcomes, clinic letters, laboratory results, radiology reports, endoscopy reports and histology was undertaken. Information on demographics, symptoms and investigation results were collected. IDA was defined by a combination of microcytosis, hypochromia and low ferritin at time of clinical review, or a diagnosis of IDA on enteral iron replacement prior to clinical review.

Results There were 163 cases of RCC in 162 patients, mean age 63.7 years, $35 \%$ female. 43 patients $(26.4 \%)$ were anaemic at diagnosis, IDA was present in 22 patients (13.5\%). $59 \%$ of IDA patients had previously undergone gastrointestinal (GI) endoscopy: one colonic melanoma; all others were normal or benign. Haematuria was reported in $22.1 \%$ of RCC patients, and $13.6 \%$ of those with IDA (3 patients). Of these 3 patients, 2 had visible haematuria and 1 had non-visible haematuria.

Conclusions Anaemia is commonly found in patients diagnosed with RCC at presentation. In our study group approximately half of anaemic patients were iron deficient. The majority of these had been previously investigated with GI endoscopy without a significant GI cause identified. Haematuria was not reported in most patients with IDA suggesting urinalysis may not be a suitable screening investigation. This adds further weight to the hypothesis that IDA associated with RCC is not from occult renal tract blood loss but from deposition in, or consumption by, the tumour itself. We propose that renal tract ultrasound is considered in all patients with IDA without significant GI pathology 


\section{PTU-121 'CHOLECYSTECTOMY' IS IT BEST SERVED HOT?}

Kristof Nemeth*, Rob Quinn, Anil Kaul. Whiston Hospital, Prescot, UK

\subsection{6/gutjil-2018-BSGAbstracts.499}

Introduction The traditional approach to the management of gallstone-related diseases and their acute exacerbations has been conservative for decades.

The long standing paradigm of endoscopic extraction of obstructing gallstones with interval cholecystectomy at the end of a cool-down period of 4 to 6 weeks has been challenged in recent years.

The National Institute for Health and Care Excellence (NICE, 2014) is recommending that patients with acute cholecystitis should have a laparoscopic cholecystectomy (LC) within a week of admission and the International Association of Pancreatology (IAP, 2013) recommends patients with gallstone pancreatitis to have LC on index admission.

Methods At our hospital we have established a hot gallbladder' service in addition to our emergency operating list with once a week dedicated sessions for patients presenting with symptomatic gallstones.

A prospective database of all 'hot' patients is maintained and we have carried out a safety and feasibility study of our 2017 cohort of patients.

Results In 2017409 cases with a median age of 58 year (range: 15 to 97) were referred to our department with hard evidence of gall stone disease.

In terms of 'hot gallbladder' cases, 117/409 were deemed suitable for the hot gallbladder list, but 19/117 patients declined surgery.

Out of the 98/117 patients 60/98 same admission LCs were performed with good results.

The median age of patients was 45 years (range: 20 to 97). The Male: Female Ratio was 1:2.5

$54 / 60$ cases were completed laparoscopically, 4/60 required conversion and $1 / 60$ case was abandoned with a cholecystostomy in-situ. The number of subtotal cholecystectomies was 5/ 60.

We had no 30 day mortality and there were no biliary tract injuries.

In terms of surgical complications there was $1 / 60$ post-operative haemotoma, $1 / 60$ bile leak and 1/60 intraoperative spillage of gall stones. 3/60 patients required readmission.

The length of stay (LOS) for the 'hot gallbladder' cohort was median 6 days (range: 0 to 17). The median length of time between onset of symptoms and presentation to hospital was 1 day (range: 0 to 21 days) and obtaining ultrasound scans took median 1 day (range: 0 to 3 ). The median waiting time for magnetic resonance cholangiopancreatography (MRCP) was 3 days from admission (range: 1 to 7 ).

In contrast the median LOS for all gall-stone related admissions in the same period were 6 days with range 0 to 56 days.

Conclusion Our experience of managing gallstone disease with prompt cholecystectomy during the same admission shows that this approach provides safe and cost-effective patient care.

In order to improve efficiency we are actively working on establishing further 'hot gall bladder' lists during the working week and reduction of waiting times for imaging is desirable.

\section{PTU-122 ACUTE UPPER GASTROINTESTINAL BLEEDING MANAGEMENT: A MULTI-CENTRE, TRAINEE LED AUDIT IN NORTH-WEST ENGLAND}

Kirsty Nixon*, Katherine White, GasTRINNoW Collaborators. Gastroenterology Trainee Research and Improvement Network North West, Manchester

\subsection{6/gutjnl-2018-BSGAbstracts. 500}

Background Despite advances in diagnostics and therapy, acute upper gastrointestinal bleeding (AUGIB) is associated with10\% mortality. The National Institute for Health and Care Excellence clinical guideline (NICE ) key priorities for implementation. Using the newly formed Gastroenterology Trainee Research and Improvement Network North West (GasTRIN NoW), we aimed to obtai multi-centre data to audit the management of AUGIB.

Method A prospective multi-centre AUGIB audit was undertaken across 10 hospitals in North West England between 30/ 10/2017 26/11/2017. Il patients admitted with suspected UGIB who underwent endoscopy (OGD).tandards were0\%100\%be offered OGD within 24 hours of admission. Each centre registered the audit locally and anonymised data was pooled within excel for further analysis in R.

Results patients were included across 10 hospitals 83\% $(n=101)$ were referred from $A+E 17 \%$ via primary care. Median age was 65 years (IQR 50-77)66\% were male. 50\% $(n=60)$ were admitted during weekdays between 07:00 and 19:00. At admission, 46\% $(n=56)$ had either a Glasgow Blatchford orpre-ockall score. 32 patients were on anti-platelet, 21 on anticoagulants (warfarin, DOAC or LMWH) and 16 on NSAIDs $(13 \%), 64 \%(n=70)$ received either oral or IV PPI prior to OGD.

Fiftyfour percent of those with varices required banding or glue therapy $(n=7 / 13)$ while $12 \%(n=13 / 108)$ required therapy for non-variceal bleeding. Haemostasis was achieved in $90 \%(n=18)$. Length of stay in these patients were longer compared to those not requiring therapy (median 6 days (IQR: $5-7$ ) vs. 4 days (IQR:2-7); p=0.04). There were 7 deaths at 30 day follow up only 1 directly attributable to AUGIB.

Conclusion NICE standards comparable to data presented by The GARnet Improvements are needed to deliver NICE standards

\section{PTU-123 EFFECTIVENESS OF A DIETETIC-LED IBS SERVICE IN THE MANAGEMENT OF PATIENTS OVER 45 YEARS}

Claire Oldale*, Natasha Cook, Alexandra Di Mambro. Glos Hospitals NHS Foundation Trust, Cheltenham, UK

\subsection{6/gutjnl-2018-BSGAbstracts.501}

Introduction The low FODMAP diet is known to be effective in improving symptoms in approx. $70 \%$ of those with Rome criteria IBS. Whilst the majority of IBS patients are young adults, there is a cohort of patients over the age of 45 with IBS who may benefit from similar interventions. In January 2017, the dietetic-led refractory IBS (RIBS) Service in Gloucestershire expanded its direct access inclusion criteria to allow GP referral of patients $>45$ years with negative GI investigation within the previous 5 years and no change in symptoms. Consultant referrals following negative investigation in this age group are also accepted. 
Methods This is a retrospective analysis of data collected during patient consultations. Patients over 45 years referred to the RIBS service by GP or consultant were assessed and provided with detailed advice on a low FODMAP diet by a specialist dietitian, to be followed for 6 weeks. An 11 point symptom evaluation was completed before and after dietary manipulation and total scores were calculated. Typical bowel frequency and stool consistency were recorded, along with responses to a global symptom question 'do you currently have satisfactory relief of your gut symptoms?'

Results 107 patients $>45$ years of age have completed treatment within the RIBS service $(82 \%$ with diarrhoea or mixed symptom predominance). Individual and total symptom scores demonstrate an average reduction in severity of almost $50 \%$ after following a FODMAP exclusion diet (figure 1), with mean total symptom score before treatment 60.9 (range 27$89 / 110$ ) reducing to 36.0 after treatment (range 11-89/110). Sixty-seven percent (72/107) of patients reported satisfactory relief of their gut symptoms following dietary restriction (figure 2). Direct-access patients who did not respond to treatment were discussed within a consultant-led MDT, with further advice, clinician review or investigation arranged as appropriate. No known cases of IBD or cancer have been missed to date using this pathway of care.

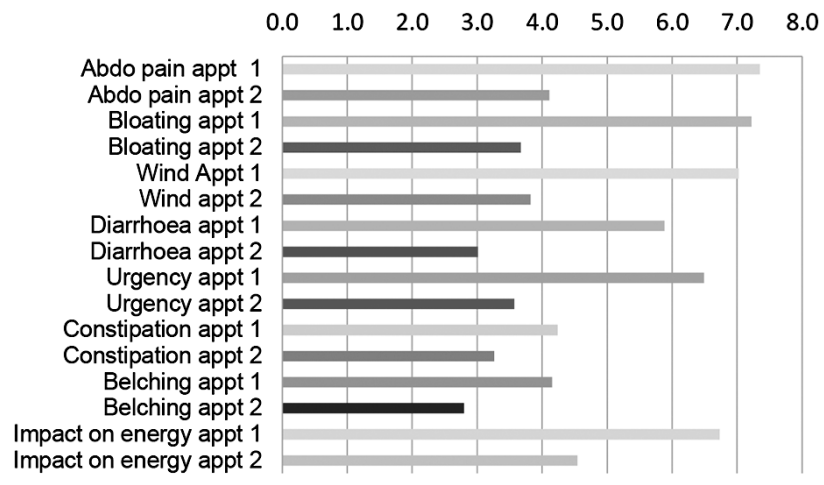

Abstract PTU-123 Figure 1 Pre (appt 1) and post (appt 2) treatment symptom scores

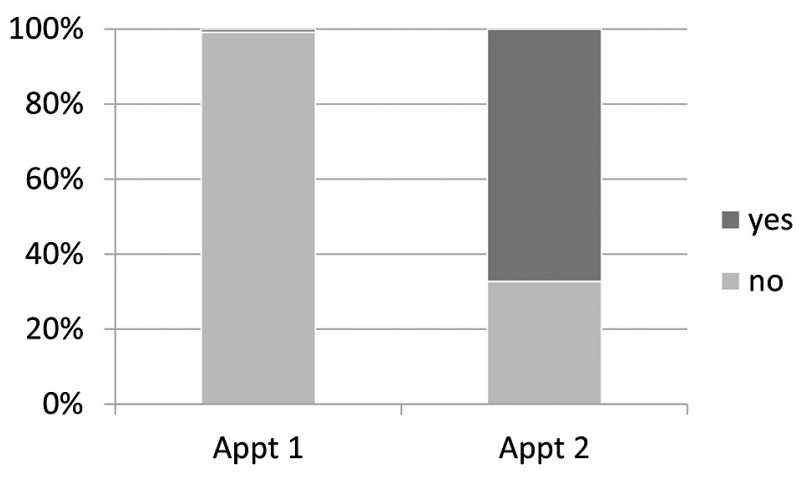

Abstract PTU-123 Figure 2 Do you currently have satisfactory relief of your gut symptoms?

Conclusions Treatment within a dietetic-led, direct-access service with an appropriate, policed care pathway and MDT support is a safe and clinically effective management strategy for patients over the age of 45 with diagnosed IBS symptoms.
The pathway provides an important adjunct to 'straight to test' referral protocols for GP's in those over 45 with stable symptoms and previously negative colonoscopy, diverting these patients from unnecessary specialist review and costly further investigation.

\section{PTU-124 REAL WORLD TOLERABILITY \& EFFICACY OF ORAL FERRIC MALTOL (FERACCRU) IN IBD ASSOCIATED ANAEMIA}

${ }^{1}$ Philip Oppong*, ${ }^{2}$ Silvia Lovato, ${ }^{1}$ Ayesha Akbar. St Mark's Hospital, Harrow, UK ${ }^{2}$ Ealing Hospital, UK

\subsection{6/gutjnl-2018-BSGAbstracts.502}

Introduction Anaemia is common in inflammatory bowel disease (IBD). Quality of life can be improved with anaemia correction. ${ }^{1}$ Conventional oral iron therapies are poorly tolerated in IBD patients. ${ }^{2}$ European Crohn's and Colitis Organisation (ECCO) guidelines suggest intravenous (IV) replacement in patients with previous intolerance to oral iron. ${ }^{3}$

Feraccru, a new oral iron complex, is cheaper than IV iron and does not require adminsitraton in hospital. In a phase 3 randomised placebo controlled trial it improved mean haemoglobin $(\mathrm{Hb})$ by $2.25 \mathrm{~g} / \mathrm{dL}$ at week 12 in patients whom previous oral ferrous products had failed. We assessed the tolerability and efficacy of Feraccru in the real world.

Methods Patients over 18 with IBD who were prescribed Feraccru between 1 February and 31 October 2017 at the London Northwest Healthcare NHS Trust were identified prospectively from prescribing clinicians and dispensary records. Data was collected from medical records and patients were contacted at least 1 month after prescription of Feraccru to assess tolerability.

Results 28 patients (16 ulcerative colitis, 12 Crohn's) were prescribed Feraccru during the study. 17/28 had taken other oral iron supplements in the past 2 years of whom 12/17 had adverse effects, the commonest being abdominal pain.

14/21 patients tolerated Feraccru (7 not contactable). 3/14 that tolerated Ferracru stopped it early due to ongoing IBD symptoms. Of the $7 / 21$ that did not tolerate it, no serious adverse events were noted; the most common side effect was abdominal pain. 5/10 patients who did not tolerate conventional oral iron tolerated Feraccru (2 not contactable).

Median baseline $\mathrm{Hb}$ was $110 \mathrm{~g} / \mathrm{dL}$ (IQR 105-127) and ferritin $14 \mathrm{mcg} / \mathrm{L}$ (IQR 12-21). 20/28 had post Feraccru bloods with a median of 114 days (IQR 92-144). between blood tests. Median post Feraccru $\mathrm{Hb}$ was $122 \mathrm{~g} / \mathrm{dL}$ (IQR 116-134) and ferritin 28mcg/L (IQR 13-43). $\mathrm{Hb}$ incremented in 16/20; median increment $14 \mathrm{~g} / \mathrm{dL}$ (IQR 8-19). $\mathrm{Hb}$ fell in 4/20; median fall $17.5 \mathrm{~g} / \mathrm{dL}$ (IQR 7.8-27.3). 6/22 had IV iron up to 4 months after their last Ferracru dose.

Conclusions Feraccru was prescribed for IBD patients with mild iron deficiency anaemia. It was tolerated in $67 \%$ of all patients and in 50\% of patients who were intolerant of conventional oral iron preparations. Hb incremented in most. Feraccru may be considered as a safe and cost effective alternative to IV iron in patients with mild IDA. Larger real life patient cohorts need to be studied, and disease activity should also be a consideration.

\section{REFERENCES}

1. Wells CW, et al. Inflamm Bowel Dis 2006;12:123-30.

2. Lugg S, et al. J Crohn's Colitis. 2014;8:876-880. 